\title{
EVALUATION OF FLAG LEAF PHYSIOLOGICAL TRAITS OF TRITICALE GENOTYPES UNDER EASTERN MEDITERRANEAN CONDITIONS
}

\author{
Rukiye KARA \\ East Mediterranean Transitional Zone Agricultural Research Institute, Kahramanmaras, TURKEY \\ Corresponding author: rukiye.kara@gthb.gov.tr
}

Received: 10.02.2016

\begin{abstract}
The primary objective of the present study is to determine the variations for physiological traits of triticale genotypes. Experiments were carried out in randomized complete block design with 3 replications during the cropping years of 2011-2012 and 2012-2013. Totally 5 cultivars and 20 lines of triticale were used as the plant material of the experiments. The relationships of grain yield with net photosynthesis rate (Pn), stomatal conductance (gs), internal $\mathrm{CO}_{2}$ concentration/ambient $\mathrm{CO}_{2}$ ratio $(\mathrm{Ci} / \mathrm{Ca})$, mesophyll conductance $(\mathrm{Mc})$, transpiration efficiency (TE), transpiration rate (Tr) and the leaf temperature (TI) were assessed through correlation and biplot analyses. For all three growth stages (booting, anthesis, grain filling), significant positive correlations were observed between Tr and Tl; gs and Tr; gs and Pn; gs and $\mathrm{Ci} / \mathrm{Ca}$ and $\mathrm{Mc}$ and Pn, and the greatest negative correlations were observed between TE and Tl. Current findings were not able indicate a single physiological trait has significant correlations with grain yield in all growth stages. However, significant negative correlations were observed between grain yield and Tl-Tr at booting and anthesis stages. It was concluded that low temperature, high net photosynthesis rate, high $\mathrm{Ci} / \mathrm{Ca}$ and low transpiration rate might be used as reliable selection criteria in further triticale breeding programs.
\end{abstract}

Keywords: Correlation, flag leaf, gas exchange parameters, grain yield, triticale

\section{INTRODUCTION}

Triticale is a cross of wheat (Triticum spp.) and rye (Secale spp.). It is an important small grain crop. Triticale has higher tolerance to biotic and abiotic stresses than wheat and higher grain yield than rye (Tohver et al., 2005). Triticale can be grown in marginal lands and is especially tolerant to drought and harsh winter conditions successfully. It also has less nutrient requirement and higher disease tolerance than the other cereal crops such as wheat, barley, corn and rice. Moreover, its grain and straw yield especially under unfavorable soil and climate conditions are higher than grain and straw yield of wheat crop (Igne et al., 2007; Kaplan et al., 2015).

Triticale has been gaining an increasing significance especially because of its higher tolerance to biotic and abiotic stresses, higher straw and grain yield and multiple uses. Therefore, triticale grown area almost was doubled in Europe during the last decade (Faostat, 2010). Several studies have been carried out in many countries to develop superior triticale varieties. It was reported that there were spring and winter triticale genotypes for grain and biomass production (Santiveri and Romagosa, 2004; Kozak et al., 2007; Lekgari et al., 2008; Bilgili et al., 2009). On the other hand, Gowda et al.
(2011) reported that most of current triticale cultivars with high grain yield tend to be poor for biomass yield, whereas the excellent forage cultivars tend to be poor for grain yield. They also indicated that based on the developed regression model, at least during earlier stages of selection, field testing could be carried out for grain yield and related traits to select potential genotypes with high biomass yield.

As mentioned above, triticale generally provides more grain and straw yields than wheat in both favorable and unfavorable growing conditions. The higher yield of triticale has been attributed to earlier stem elongation stage, longer spike formation period (Lopez-Castaneda and Richards, 1994; Giunta et al., 2001) and higher spike fertility (Giunta et al., 2001, 2003). It was also shown that triticale produced fewer tillers, had greater early vigour levels (Lopez-Castaneda and Richards, 1994; Giunta et al., 2003) and larger root system at early growth stages (Richards et al., 2007). Simulations performed by Bassu et al., (2011) showed that the highest levels in wheat could be achieved through increasing transpiration-water use efficiency. But they also suggested that early vigour, remobilization of stem carbohydrates to grains and early root growth also contributed positively to a yield increase in different growing environments. 
Triticale can remobilize more assimilates, which have been accumulated prior to grain filling period, to grains (Lopez-Castaneda and Richards, 1994; Ruuska et al., 2006). The net photosynthetic rate differences of wheat cultivars at high temperatures have been found to be associated with lower leaf chlorophyll concentration and changes of chlorophyll a:b ratio caused by accelerated leaf senescence (Al-Khatib and Paulsen, 1984; Harding et al., 1990).

Measurement of whole canopy carbon exchange rate in a very short time is generally an expensive method and several internal and external factors can simultaneously affect photosynthetic rate throughout growing period of crop. Therefore, it is suggested that in order to relate accurately crop productivity to leaf photosynthesis, quantification of canopy photosynthesis is required (Reynolds et al., 2000). Scientists measured net photosynthesis rate, stomatal conductance, chlorophyll content and dark respiration rate of 16 wheat cultivars and indicated that differences in net photosynthesis rate throughout the crop cycle as well as variation in onset of senescence might be important variables affecting wheat yield potential in warm environments, and net photosynthetic rate during the grain filling period was also strongly associated with chlorophyll loss (Reynolds et al., 2000). Hura et al., (2009) aimed to determine the differences in the activity of the photosynthesis apparatus in 10 genotypes of winter triticale grown under optimal conditions and to determine whether or not such measurements could provide a correlation that explains the usefulness of photosynthetic parameters in the estimation of harvest and growth of plants. They found out that there were significant correlations between the yield and some parameters of chlorophyll fluorescence and indicated that leaf gas exchange and parameters of chlorophyll fluorescence were useful for estimation of the functional state of the photosynthetic apparatus and could be selection criteria in plant breeding.

The present research was conducted to evaluate the relationships between grain yield and flag leaf physiological traits (net photosynthesis rate, stomatal conductance, intercellular $\mathrm{CO}_{2}$ concentration, ambient $\mathrm{CO}_{2}$ concentration, transpiration rate, mesophyll conductance, transpiration efficiency and leaf temperature) of triticale genotypes grown under Eastern Mediterranean conditions.

\section{MATERIALS AND METHODS}

Experiments were carried out in the research fields of Eastern Mediterranean Transition Zone Agricultural Research Center during the growing seasons of 2011-12 and $2012-13$. The genotypes used as material in experiments are provided in Table 1.

Table 1. Triticale genotypes used in the experiments and their pedigrees

\begin{tabular}{ll}
\hline Genotypes & Pedigrees \\
\hline Line 1 & MIKHAM-2002 / 01-02 STBVD-21 \\
Line 2 & CIMMYT-3 / ANOAS_3/TATU_4//SUSI_2 \\
Line 3 & 431_TU_1-11/3/DARGO/IBEX//CIVET\#2/KARMA \\
Line 4 & SAMUR SORTU / 01-02 STBVD-19 \\
Cultivar 1 & TATLICAK-97 \\
Line 6 & CIMMYT-3 / KARMA \\
Line 7 & 01-02 KTBVD-1/ KARMA \\
Line 8 & 23FAHAT5/POLLMER3CTSS/POLLMER_3/FOCA_2-1 \\
Line 9 & 23FAHAT5/POLLMER3CTSS/POLLMER_3/FOCA_2-1 \\
Cultivar 2 & MELEZ-2001 \\
Line 11 & 23FAHAT5/POLLMER3CTSS/POLLMER_3/FOCA_2-1 \\
Line 12 & BAGAL_3/FARAS_1/3/ARDI_1/TOPO1419//ERIZO_9/KARMA \\
Line 13 & FAHAD_8-2*2//PTR/PND-T/3/ERIZO_11//YOGUI_3/ POLLMER_3/FOCA_2-1 \\
Line 14 & CT179.80/3/150.83//2*TESMO_1MUSX603/01-02KTVD-17 \\
Cultivar 3 & MIKHAM-2002 \\
Line 16 & CIMMYT-3 / ANOAS_3/TATU_4//SUSI_2 \\
Line 17 & CIMMYT-3 / KARMA \\
Line 18 & 23FAHAT5/POLLMER3CTSS/POLLMER_3/FOCA_2-1 \\
Line 19 & CHD1089/POLLMER_2.3.1/POLLMER_3/FOCA_2-1 \\
Cultivar 4 & ALPERBEY \\
Line 21 & CT179.80/3/150.83//2*TESMO_1MUSX603/01-02KTVD-17 \\
Line 22 & PRESTO 6D(6A)//BULL_10/MANATI_1/01-02 KTVD-32 \\
Line 23 & BULL_10/MANATI_1//FARAS/CMH84.4414 \\
Line 24 & 33--1/42-2 \\
Cultivar 5 & KARMA-2000 \\
\hline
\end{tabular}

The research province, Kahramanmaras is located in Eastern-Mediterranean Region between $37^{\circ} 38^{\prime}$ North latitudes and $36^{\circ} 37^{\prime}$ 'East longitudes and has an altitude of
$568 \mathrm{~m}$. Mediterranean climate is dominant in the province and day-night temperature difference is low. The Mediterranean climate is typical of the region and some 
climatic data are given in Table 2. Some chemical and 0-30 $\mathrm{cm}$ topsoil are given in Table 3. physical traits of two years experiment soil sampled from

Table 2. Climate parameters for experimental years and long term averages

\begin{tabular}{lccccccccc}
\hline & \multicolumn{3}{c}{ Precipitation (mm) } & \multicolumn{3}{c}{ Temperature $\left(^{\circ} \mathbf{C}\right)$} & \multicolumn{3}{c}{ Relative Humidity (\%) } \\
\hline \multirow{2}{*}{ Months } & $2011-$ & $2012-$ & Long Term & $2011-$ & $2012-$ & Long & $2011-$ & $2012-$ & Long \\
& 2012 & 2013 & $(1975-2011)$ & 2012 & 2013 & Term & 2012 & 2013 & Term \\
\hline November & 93.2 & 36.4 & 90.9 & 8.7 & 13.4 & 11.5 & 60.6 & 70.6 & 64.7 \\
December & 85.2 & 67.6 & 124.4 & 6.3 & 7.7 & 6.6 & 64.7 & 76.4 & 71.3 \\
January & 325.0 & 111.0 & 125.4 & 6.9 & 6.2 & 4.9 & 79.9 & 72.3 & 70.0 \\
February & 199.1 & 131.9 & 112.3 & 4.1 & 8.6 & 6.3 & 61.9 & 74.0 & 66.0 \\
March & 0.0 & 77.5 & 94.8 & 8.6 & 11.3 & 10.6 & 51.8 & 52.1 & 60.5 \\
April & 0.0 & 65.9 & 76.1 & 17.7 & 17.1 & 15.4 & 49.3 & 52.5 & 58.4 \\
May & 41.3 & 76.5 & 39.3 & 19.9 & 22.4 & 20.4 & 55.8 & 53.4 & 54.7 \\
June & 13.0 & 16.3 & 5.9 & 27.9 & 25.4 & 25.2 & 33.4 & 43.9 & 50.7 \\
\hline Total & 756.8 & 583.1 & 669.1 & & & & & & \\
\hline Mean & & & & 12.5 & 14.0 & 12.6 & 57.2 & 61.9 & 62.0 \\
\hline
\end{tabular}

Table 3. Physical and chemical characteristics of experimental soils

\begin{tabular}{llccccc}
\hline Years & Texture & $\mathbf{p H}$ & $\mathbf{C a C O}_{3}(\boldsymbol{\%})$ & $\begin{array}{c}\mathbf{P}_{2} \mathbf{O}_{5} \\
\left(\mathbf{k g ~ h a}^{-\mathbf{1}}\right)\end{array}$ & $\begin{array}{c}\mathbf{K}_{2} \mathbf{O} \\
\left(\mathbf{k g ~ h a}^{-\mathbf{1}}\right)\end{array}$ & $\begin{array}{c}\text { Organic matter } \\
(\%)\end{array}$ \\
\hline $2011-12$ & Loamy & 7.61 & 12.55 & 0.46 & 4.59 & 1.22 \\
$2012-13$ & Loamy & 8.00 & 24.59 & 0.80 & 12.70 & 0.97 \\
\hline
\end{tabular}

Experiments were carried out in randomized complete block design with 3 replicated. Seeding rate was 500 seeds $\mathrm{m}^{-2}$ and seeding was performed with a plot-drill over $6 \mathrm{x}$ $1.5 \mathrm{~m}$ size plots. There were 6 rows in each plot with row spacing of $20 \mathrm{~cm}$. Sowing dates were on 5 December 2011 and 3 January 2013; harvesting date were 20 June for each two years. In both years, $80 \mathrm{~kg} \mathrm{~N}$ and $80 \mathrm{~kg} \mathrm{P}_{2} \mathrm{O}_{5} \mathrm{ha}^{-1}$ were applied to soil during sowings and additional $100 \mathrm{~kg} \mathrm{ha}^{-1}$ $\mathrm{N}$ was supplied during tillering period. Irrigation was not performed in both years and herbicide with active ingredient of Tribenuron-Methyl was used for broad-leaf weeds.

During the booting, anthesis and grain filling stages, clear sunny days were selected for gas-exchange measurements, namely: net photosynthesis rate (Pn), stomatal conductance (gs), intercellular $\mathrm{CO}_{2}$ concentration (Ci), ambient $\mathrm{CO}_{2}$ concentration $(\mathrm{Ca})$, transpiration rate (Tr) and leaf temperature (Tl) using a portable photosynthesis system (LCpro+ Portable Gas Analyser). The measurements were conducted under sunlight between 9:00 and 12:00 and the leaves were maintained at right angles with respect to incident solar radiation. At least five different flag leaves were measured per genotype for each plot on a single day. Due to phonological differences among the cultivars, three approximate growth stages were designated as booting, anthesis and grain filling periods for measurements in all cultivars. The flag leaf transpiration efficiency (TE) was calculated as: $\mathrm{TE}=\mathrm{Pn} / \mathrm{Tr}$. Internal $\mathrm{CO}_{2}$ concentration/ambient $\mathrm{CO}_{2}$ ratio $(\mathrm{Ci} / \mathrm{Ca})$ were estimated from gas exchange measurements using the equations developed by von Caemmerer and Farquhar (1981). The apparent leaf mesophyll conductance $(\mathrm{Mc}=\mathrm{Pn} / \mathrm{Ci})$ was also calculated.

Correlations analyses were performed by using SAS (SAS Inst., 1999) statistical software. The GT biplot is generated by plotting standardized data of physiological traits in different stages of triticale respectively, so that each genotype or trait is represented by a marker in the biplot (Yan and Kang, 2003). In the GT biplot, a vector is drawn from the biplot origin to each marker of the traits to facilitate visualization of the relationships between and among the traits generated by Excel Macro (Lipkovich and Smith, 2002).

\section{RESULTS AND DISCUSSIONS}

Two years experimental results revealed significant differences in all traits except for gs, $\mathrm{Ci} / \mathrm{Ca}$ and $\mathrm{Tr}$ of the years $(P<0.01)$, in TE of genotypes $(P<0.01)$ and in Tl, Tr, gs, Pn, Mc and TE of the growth stages (Table 4, 5 and 6).

The differences in grain yields of genotypes were found to be significant $(\mathrm{P} \leq 0.01)$. Grain yield values under rain-fed conditions varied between $4258-6519 \mathrm{~kg} / \mathrm{ha}$ with the greatest yield in Line 19 genotype and the lowest yield in Line 9 genotype (Table 7). The differences in grain yields of the years were mainly resulted from differences in precipitations of the years. Lack of precipitations especially in March and April (with rapid developments in plants) of the first year and high precipitations of the second year ultimately affected the grain yields resulted in significant differences in grain yields of the years. 
Table 4. Mean values for leaf temperature (Tl), stomatal conductance (gs), net photosynthesis rate $(\mathrm{Pn})$, internal $\mathrm{CO}_{2}$ concentration/ambient $\mathrm{CO}_{2}$ ratio $(\mathrm{Ci} / \mathrm{Ca})$, mesophyll conductance $(\mathrm{Mc})$, transpiration rate $(\mathrm{Tr})$ and transpiration efficiency (TE) during booting stage

\begin{tabular}{|c|c|c|c|c|c|c|c|}
\hline Genotypes & $\begin{array}{c}\mathrm{Tl} \\
\left({ }^{0} \mathrm{C}\right)\end{array}$ & $\begin{array}{c}g S \\
\left(\mathrm{~mol} \cdot \mathrm{m}^{-2} \mathrm{~s}^{-1}\right)\end{array}$ & $\begin{array}{c}\text { Pn } \\
\left(\mu \mathrm{mol} \cdot \mathrm{m}^{-2} \mathrm{~s}^{-1}\right)\end{array}$ & $\mathrm{Ci} / \mathrm{Ca}$ & \multicolumn{2}{|c|}{ 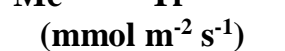 } & $\begin{array}{c}\mathrm{TE} \\
\left(\mathrm{mmol} \mathrm{mol}^{-1}\right)\end{array}$ \\
\hline Line 1 & 33.80 & 0.320 & 17.15 & 0.590 & 76.16 & 6.232 & 3.304 \\
\hline Line 2 & 32.65 & 0.320 & 18.84 & 0.580 & 82.38 & 5.793 & 3.945 \\
\hline Line 3 & 33.92 & 0.340 & 19.14 & 0.580 & 84.48 & 6.318 & 3.358 \\
\hline Line 4 & 34.23 & 0.310 & 18.76 & 0.560 & 85.56 & 6.257 & 3.287 \\
\hline Tatlicak-97 & 34.15 & 0.380 & 20.38 & 0.570 & 91.35 & 6.837 & 3.439 \\
\hline Line 6 & 34.32 & 0.350 & 19.96 & 0.560 & 91.16 & 6.855 & 3.503 \\
\hline Line 7 & 34.15 & 0.362 & 19.80 & 0.567 & 89.34 & 6.665 & 3.458 \\
\hline Line 8 & 33.97 & 0.310 & 18.67 & 0.561 & 84.56 & 5.978 & 3.602 \\
\hline Line 9 & 34.37 & 0.343 & 19.41 & 0.558 & 88.18 & 6.410 & 3.564 \\
\hline Melez-2001 & 34.52 & 0.402 & 18.65 & 0.604 & 77.73 & 7.135 & 3.182 \\
\hline Line 11 & 35.52 & 0.345 & 18.84 & 0.584 & 82.25 & 7.317 & 3.018 \\
\hline Line 12 & 34.33 & 0.297 & 18.47 & 0.560 & 84.34 & 6.363 & 3.517 \\
\hline Line 13 & 34.40 & 0.402 & 21.71 & 0.578 & 94.52 & 7.632 & 3.663 \\
\hline Line 14 & 35.12 & 0.340 & 18.67 & 0.584 & 82.15 & 7.075 & 3.017 \\
\hline Mikham-2002 & 34.40 & 0.330 & 19.65 & 0.568 & 86.33 & 6.992 & 3.594 \\
\hline Line 16 & 35.25 & 0.295 & 17.84 & 0.580 & 77.25 & 6.843 & 2.945 \\
\hline Line 17 & 35.57 & 0.348 & 18.69 & 0.591 & 81.23 & 7.720 & 2.962 \\
\hline Line 18 & 36.05 & 0.318 & 19.76 & 0.545 & 91.92 & 7.548 & 3.041 \\
\hline Line 19 & 34.63 & 0.365 & 18.43 & 0.575 & 82.63 & 7.048 & 2.942 \\
\hline Alperbey & 35.93 & 0.380 & 20.44 & 0.557 & 91.32 & 7.863 & 2.983 \\
\hline Line 21 & 34.60 & 0.330 & 19.99 & 0.557 & 91.27 & 7.087 & 3.463 \\
\hline Line 22 & 35.75 & 0.392 & 20.52 & 0.579 & 89.34 & 8.395 & 2.910 \\
\hline Line 23 & 34.83 & 0.320 & 19.13 & 0.573 & 83.49 & 6.952 & 3.216 \\
\hline Line 24 & 35.32 & 0.352 & 18.55 & 0.578 & 81.16 & 7.540 & 2.777 \\
\hline Karma-2000 & 35.88 & 0.350 & 19.45 & 0.574 & 86.39 & 7.827 & 2.954 \\
\hline Mean & 34.71 & 0.344 & 19.24 & 0.573 & 85.46 & 6.987 & 3.149 \\
\hline Mean of 2012 & 38.74 & 0.344 & 17.60 & 0.579 & 78.28 & 9.012 & 2.056 \\
\hline Mean of 2013 & 30.67 & 0.344 & 20.87 & 0.566 & 91.84 & 4.963 & 4.476 \\
\hline LSD & 2.32 & 0.11 & 2.94 & 0.06 & 17.4 & 1.74 & 0.98 \\
\hline $\mathrm{CV}$ & 5.83 & 28.8 & 13.4 & 9.31 & 17.8 & 21.7 & 26.1 \\
\hline Year & $* *$ & ns & $* *$ & ns & $* *$ & $* *$ & $* *$ \\
\hline Genotypes & $\mathrm{ns}$ & $\mathrm{ns}$ & $\mathrm{ns}$ & $\mathrm{ns}$ & $\mathrm{ns}$ & ns & $\mathrm{ns}$ \\
\hline YearxGenotypes & $\mathrm{ns}$ & ns & ns & ns & $\mathrm{ns}$ & ns & ns \\
\hline
\end{tabular}

ns, non-significant and ${ }^{* *}$, mean significant difference at 0.01 .

Regarding the stage of development, most of the physiological traits were relatively stable between booting and anthesis. $\mathrm{Tl}, \mathrm{Ci} / \mathrm{Ca}$ and $\mathrm{TE}$ increased moderately between booting and anthesis. However, Tl, Tr, gs, Pn and Mc declined between anthesis and grain filling (Table 5 and 6). Although varied based on genotype, average $23 \%$ decrease was observed in Pn between booting and anthesis. A similar reduction in $\mathrm{Pn}$ at a later stage, between anthesis and grain filling was also observed as $6 \%$. In previous studies, strong decreases were reported in $\mathrm{Pn}$, gs ve $\mathrm{Tr}$ of wheat and barley genotypes with decreasing temperature and soil moisture (Allahverdiyev et al., 2015; Azhand et al., 2015). 
Table 5. Mean values for leaf temperature $(\mathrm{Tl})$, stomatal conductance (gs), net photosynthesis rate $(\mathrm{Pn})$, internal $\mathrm{CO}_{2}$ concentration/ambient $\mathrm{CO}_{2}$ ratio $(\mathrm{Ci} / \mathrm{Ca})$, mesophyll conductance $(\mathrm{Mc})$, transpiration rate $(\mathrm{Tr})$ and transpiration efficiency $(\mathrm{TE})$ during anthesis stage

\begin{tabular}{|c|c|c|c|c|c|c|c|}
\hline Genotypes & $\begin{array}{c}\mathrm{Tl} \\
\left({ }^{0} \mathrm{C}\right) \\
\end{array}$ & $\begin{array}{c}\text { gs } \\
\left(\mathrm{mol} \cdot \mathrm{m}^{-2} \mathrm{~s}^{-1}\right) \\
\end{array}$ & $\begin{array}{c}\text { Pn } \\
\left(\mu \mathrm{mol} \cdot \mathrm{m}^{-2} \mathrm{~s}^{-1}\right)\end{array}$ & $\mathbf{C i} / \mathbf{C a}$ & \multicolumn{2}{|c|}{$\left(\mathrm{mmol} \mathrm{m} \mathrm{m}^{-2} \mathrm{~s}^{-1}\right)$} & $\begin{array}{c}\text { TE } \\
\left(\mathrm{mmol} \mathrm{mol}^{-1}\right)\end{array}$ \\
\hline Line 1 & 36.25 & 0.190 & 13.99 & 0.535 & 68.63 & 5.243 & 2.767 \\
\hline Line 2 & 35.90 & 0.240 & 15.61 & 0.566 & 72.55 & 5.880 & 2.722 \\
\hline Line 3 & 36.20 & 0.262 & 16.25 & 0.566 & 77.20 & 6.185 & 2.635 \\
\hline Line 4 & 35.58 & 0.302 & 15.88 & 0.617 & 68.16 & 6.487 & 2.487 \\
\hline Tatlıcak-97 & 35.33 & 0.238 & 14.61 & 0.566 & 68.00 & 5.162 & 2.885 \\
\hline Line 6 & 35.63 & 0.267 & 16.60 & 0.559 & 78.78 & 5.797 & 2.872 \\
\hline Line 7 & 35.65 & 0.242 & 14.47 & 0.586 & 65.25 & 5.565 & 2.644 \\
\hline Line 8 & 35.72 & 0.227 & 13.99 & 0.561 & 67.32 & 5.275 & 2.777 \\
\hline Line 9 & 36.02 & 0.250 & 14.94 & 0.586 & 67.33 & 5.922 & 2.570 \\
\hline Melez-2001 & 36.05 & 0.233 & 14.48 & 0.582 & 65.13 & 5.795 & 2.598 \\
\hline Line 11 & 35.68 & 0.255 & 14.82 & 0.597 & 66.37 & 5.802 & 2.597 \\
\hline Line 12 & 35.73 & 0.257 & 14.48 & 0.608 & 62.28 & 5.878 & 2.525 \\
\hline Line 13 & 35.55 & 0.215 & 13.55 & 0.576 & 62.55 & 5.130 & 2.678 \\
\hline Line 14 & 35.87 & 0.238 & 15.13 & 0.569 & 55.37 & 5.655 & 2.764 \\
\hline Mikham-2002 & 35.63 & 0.242 & 14.24 & 0.601 & 62.27 & 5.595 & 2.615 \\
\hline Line 16 & 36.12 & 0.250 & 14.16 & 0.603 & 62.58 & 5.953 & 2.492 \\
\hline Line 17 & 36.00 & 0.198 & 15.82 & 0.487 & 85.26 & 5.147 & 3.234 \\
\hline Line 18 & 35.87 & 0.218 & 14.79 & 0.552 & 71.20 & 5.317 & 2.815 \\
\hline Line 19 & 36.28 & 0.252 & 15.39 & 0.568 & 71.64 & 6.025 & 2.588 \\
\hline Alperbey & 35.85 & 0.250 & 14.26 & 0.604 & 62.55 & 5.857 & 2.488 \\
\hline Line 21 & 35.93 & 0.252 & 14.65 & 0.599 & 64.05 & 5.900 & 2.505 \\
\hline Line 22 & 36.13 & 0.218 & 14.57 & 0.535 & 74.13 & 5.387 & 2.837 \\
\hline Line 23 & 35.72 & 0.242 & 15.65 & 0.559 & 73.08 & 5.697 & 2.855 \\
\hline Line 24 & 36.37 & 0.215 & 13.60 & 0.586 & 61.73 & 5.627 & 2.441 \\
\hline Karma-2000 & 35.88 & 0.267 & 14.50 & 0.615 & 61.28 & 6.127 & 2.446 \\
\hline Mean & 35.88 & 0.241 & 14.82 & 0.575 & 67.79 & 5.696 & 2.570 \\
\hline Mean of 2012 & 37.02 & 0.265 & 16.10 & 0.563 & 76.33 & 6.597 & 2.465 \\
\hline Mean of 2013 & 34.73 & 0.217 & 13.53 & 0.588 & 58.57 & 4.795 & 2.881 \\
\hline LSD & 0.87 & 0.06 & 2.08 & 0.06 & 14.6 & 0.87 & 0.39 \\
\hline $\mathrm{CV}$ & 2.11 & 22.7 & 12.3 & 9.84 & 18.8 & 13.4 & 12.6 \\
\hline Year & $* *$ & $* *$ & $* *$ & $* *$ & $* *$ & $* *$ & $* *$ \\
\hline Genotypes & ns & $\mathrm{ns}$ & $\mathrm{ns}$ & ns & ns & ns & $*$ \\
\hline YearxGenotypes & ns & ns & ns & ns & $\mathrm{ns}$ & ns & ns \\
\hline
\end{tabular}

Leaf temperature was measured as $34.7^{\circ} \mathrm{C}$ in booting stage, $35.9^{\circ} \mathrm{C}$ in anthesis and $30.9^{\circ} \mathrm{C}$ in grain filling stages. While low daily temperature in grain filling stage and during the period in which $\mathrm{Tl}$ measurements were made were similar to findings of Delgado et al., (1994) and such low temperatures resulted in low leaf temperatures in triticale genotypes. On the other hand, high temperature and relative humidity post-booting growth stage resulted in higher temperatures in $\mathrm{Tl}$. Delgado et al. (1994) carried out a study on bread wheat for two years and reported average daily maximum temperatures of the first and the second year respectively as 32 and $36{ }^{\circ} \mathrm{C}$ and resultant leaf temperatures respectively as $31.7^{\circ} \mathrm{C} 34.7^{\circ} \mathrm{C}$.
As an average of genotypes, $\operatorname{Tr}$ decreased from booting to grain filling and observed as $6.585 \mathrm{mmol} \mathrm{m}^{-2} \mathrm{~s}$ ${ }^{1}$ in the first year and $4.629 \mathrm{mmol} \mathrm{m}^{-2} \mathrm{~s}^{-1}$ in the second year. Higher precipitations were observed in May of the second year in which measurements were made than the first year. The $50 \mathrm{~mm}$ precipitation received on May 13 before the measurements resulted to have higher transpiration rates in the first year. Qui et al. (2008) indicated significant effects of irrigation intervals on transpiration rates and reported $\operatorname{Tr}$ as between 4.8-5.8 $\mathrm{mmol} \mathrm{m} \mathrm{m}^{-2} \mathrm{~s}^{-1}$ in the first year and between 7.3-8.4 mmol $\mathrm{H}_{2} \mathrm{O} \mathrm{m}^{-2} \mathrm{~s}^{-1}$ in the second year. Dry soil conditions caused a reduction in transpiration rates. Different decrease trends were observed for Tr of triticale genotypes. Similar effects were also reported for flowering and legume formation of field bean (Hura et al., 2007). 
Table 6. Mean values for leaf temperature ( $\mathrm{Tl})$, stomatal conductance (gs), net photosynthesis rate $(\mathrm{Pn})$, internal $\mathrm{CO}_{2}$ concentration/ambient $\mathrm{CO}_{2}$ ratio $(\mathrm{Ci} / \mathrm{Ca})$, mesophyll conductance $(\mathrm{Mc})$, transpiration rate $(\mathrm{Tr})$ and transpiration efficiency (TE) during grain filling stage

\begin{tabular}{|c|c|c|c|c|c|c|c|}
\hline Genotypes & $\begin{array}{c}\mathrm{Tl} \\
\left({ }^{0} \mathrm{C}\right) \\
\end{array}$ & $\begin{array}{c}\text { gS } \\
\left(\mathrm{mol} \cdot \mathrm{m}^{-2} \mathrm{~s}^{-1}\right)\end{array}$ & $\begin{array}{c}\text { Pn } \\
\left(\mu \mathrm{mol} \cdot \mathrm{m}^{-2} \mathrm{~s}^{-1}\right)\end{array}$ & $\mathrm{Ci} / \mathrm{Ca}$ & \multicolumn{2}{|c|}{ 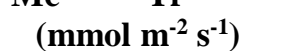 } & $\begin{array}{c}\text { TE } \\
\left(\mathrm{mmol} \mathrm{mol}^{-1}\right)\end{array}$ \\
\hline Line 1 & 30.62 & 0.205 & 14.50 & 0.585 & 60.57 & 4.172 & 3.933 \\
\hline Line 2 & 30.25 & 0.205 & 13.33 & 0.627 & 57.62 & 3.825 & 3.541 \\
\hline Line 3 & 29.57 & 0.237 & 13.79 & 0.607 & 58.32 & 3.802 & 3.984 \\
\hline Line 4 & 29.93 & 0.230 & 14.63 & 0.612 & 60.58 & 4.043 & 3.723 \\
\hline Tatlıcak-97 & 30.47 & 0.190 & 14.67 & 0.571 & 59.05 & 3.840 & 3.931 \\
\hline Line 6 & 30.95 & 0.183 & 13.13 & 0.588 & 53.68 & 3.867 & 3.502 \\
\hline Line 7 & 29.88 & 0.227 & 13.97 & 0.641 & 56.27 & 3.963 & 3.704 \\
\hline Line 8 & 30.32 & 0.215 & 13.67 & 0.644 & 54.57 & 4.048 & 3.468 \\
\hline Line 9 & 30.13 & 0.198 & 14.37 & 0.596 & 62.03 & 3.947 & 3.846 \\
\hline Melez-2001 & 31.47 & 0.165 & 13.63 & 0.569 & 59.58 & 3.987 & 3.501 \\
\hline Line 11 & 31.68 & 0.200 & 14.06 & 0.610 & 58.27 & 4.413 & 3.285 \\
\hline Line 12 & 30.78 & 0.188 & 13.76 & 0.608 & 59.13 & 3.905 & 3.537 \\
\hline Line 13 & 30.98 & 0.223 & 13.78 & 0.616 & 56.62 & 4.097 & 3.472 \\
\hline Line 14 & 30.58 & 0.203 & 14.52 & 0.609 & 63.47 & 4.072 & 3.728 \\
\hline Mikham-2002 & 31.05 & 0.180 & 14.22 & 0.581 & 62.29 & 3.960 & 3.662 \\
\hline Line 16 & 30.68 & 0.193 & 13.16 & 0.625 & 54.47 & 3.947 & 3.418 \\
\hline Line 17 & 31.98 & 0.218 & 14.88 & 0.608 & 60.45 & 4.757 & 3.226 \\
\hline Line 18 & 32.03 & 0.198 & 14.63 & 0.607 & 58.53 & 4.708 & 3.210 \\
\hline Line 19 & 31.27 & 0.213 & 13.64 & 0.625 & 52.45 & 4.353 & 3.222 \\
\hline Alperbey & 31.82 & 0.185 & 12.92 & 0.591 & 55.05 & 4.180 & 3.200 \\
\hline Line 21 & 30.53 & 0.193 & 14.16 & 0.537 & 75.00 & 3.775 & 3.893 \\
\hline Line 22 & 31.87 & 0.210 & 13.84 & 0.641 & 57.68 & 4.910 & 2.926 \\
\hline Line 23 & 31.45 & 0.163 & 12.45 & 0.581 & 51.27 & 3.715 & 3.589 \\
\hline Line 24 & 31.05 & 0.202 & 14.30 & 0.620 & 61.54 & 4.352 & 3.426 \\
\hline Karma-2000 & 32.00 & 0.228 & 14.21 & 0.629 & 61.29 & 4.823 & 3.042 \\
\hline Mean & 30.93 & 0.202 & 13.93 & 0.605 & 58.79 & 4.138 & 3.519 \\
\hline Mean of 2012 & 32.12 & 0.148 & 13.21 & 0.563 & 61.33 & 4.147 & 3.354 \\
\hline Mean of 2013 & 29.75 & 0.257 & 14.64 & 0.647 & 55.27 & 4.130 & 3.684 \\
\hline LSD & 1.73 & 0.06 & 1.49 & 0.07 & 13.2 & 0.85 & 0.79 \\
\hline $\mathrm{CV}$ & 4.87 & 27.4 & 9.31 & 9.44 & 19.7 & 17.91 & 19.8 \\
\hline Year & $* *$ & $* *$ & $* *$ & $* *$ & $* *$ & $\mathrm{~ns}$ & $* *$ \\
\hline Genotypes & $\mathrm{ns}$ & $\mathrm{ns}$ & $\mathrm{ns}$ & $\mathrm{ns}$ & $\mathrm{ns}$ & $\mathrm{ns}$ & $\mathrm{ns}$ \\
\hline YearxGenotypes & ns & ns & ns & ns & $\mathrm{ns}$ & ns & ns \\
\hline
\end{tabular}

ns, non-significant and $* *$, mean significant difference at 0.01 .

Stomatal conductance was measured as $0.252 \mathrm{~mol} \mathrm{H}_{2} \mathrm{O}$ $\mathrm{m}^{-2} \mathrm{~s}^{-1}$ in the first year and $0.272 \mathrm{~mol} \mathrm{H}_{2} \mathrm{O} \mathrm{m} \mathrm{m}^{-2} \mathrm{~s}^{-1}$ in the second year (data not shown). Compared to the second year, gs values decreased in the first year because of insufficient precipitations in grain filling period. Therefore, under insufficient moisture conditions, stomatal closure to prevent water loss and consequent decreases in gs were reported (Cornic, 2000). Ethylene amount and ABA synthesis in plants are increasing under drought conditions. Thus, stomata are closing and senescence of leaves is accelerating. Therefore, stomatal conductance is decreasing. Also lower minimum temperatures of the first year at the time of measurements decreased gs values. It was reported that stomatal conductance was influenced by minimum temperatures and low night temperatures reduced gs and $\mathrm{Ci}$ values (Bunce, 1998). As the average of genotypes, gs values gradually decreased from booting to grain filling stage.
Delgado et al. (1994) reported in wheat that gs value of booting, anthesis and grain filling stages respectively as $0.678,0.586$ and $0.317 \mathrm{~mol} \mathrm{H}_{2} \mathrm{O} \mathrm{m}^{-2} \mathrm{~s}^{-1}$; Reynolds et al. (2000) reported the values respectively as $0.680,0.590$ and $0.320 \mathrm{~mol} \mathrm{H}_{2} \mathrm{O} \mathrm{m}^{-2} \mathrm{~s}^{-1}$ and indicated decreasing gs values with aging of the leaves. Boutraa et al. (2015) compared gs values under $20{ }^{\circ} \mathrm{C}$ and reported $28 \%$ decrease under moderate temperatures and $46 \%$ decrease under high temperatures. Additionally, compared species differed in gs, particularly in pre-anthesis period in which triticale showed a stomatal conductance markedly higher than durum wheat. The difference in stomatal conductance between two species persisted after anthesis, although only in the irrigated treatment. Under rainfed conditions, on the contrary, initially higher stomatal conductance of triticale decreased to values very similar to those of durum wheat (Motzo et al., 2013). 
Table 7. The values of grain yield $(\mathrm{kg} / \mathrm{ha})$ of triticale genotypes

\begin{tabular}{|c|c|c|c|c|c|}
\hline \multirow{2}{*}{$\begin{array}{l}\text { Genotypes } \\
\text { Line } 1\end{array}$} & \multicolumn{2}{|c|}{ 2011-12 } & \multirow{2}{*}{$\begin{array}{c}\mathbf{2 0 1 2 - 1 3} \\
6688.3\end{array}$} & \multicolumn{2}{|c|}{ Mean } \\
\hline & 6016.3 & abcd & & 6352.3 & $a b$ \\
\hline Line 2 & 5790.7 & abcd & 6790.0 & 6290.3 & $a b c$ \\
\hline Line 3 & 5126.7 & bcdefg & 6508.7 & 5817.7 & $a b c$ \\
\hline Line 4 & 5708.3 & abcde & 7141.0 & 6424.7 & $\mathrm{a}$ \\
\hline Tatlicak-97 & 4115.0 & $\mathrm{fg}$ & 5176.7 & 4645.8 & de \\
\hline Line 6 & 5481.7 & abcde & 7460.7 & 6471.2 & $\mathrm{a}$ \\
\hline Line 7 & 5738.3 & abcde & 6517.7 & 6128.0 & $a b c$ \\
\hline Line 8 & 5884.3 & abcd & 6329.7 & 6107.0 & $a b c$ \\
\hline Line 9 & 3933.0 & $\mathrm{~g}$ & 4582.3 & 4257.7 & $\mathrm{e}$ \\
\hline Melez-2001 & 4487.7 & efg & 6111.7 & 5299.7 & $\mathrm{~cd}$ \\
\hline Line 11 & 5236.3 & $\mathrm{abc}$ & 6710.3 & 6473.3 & $\mathrm{a}$ \\
\hline Line 12 & 5797.0 & abcd & 7087.0 & 6442.0 & $\mathrm{a}$ \\
\hline Line 13 & 6126.3 & $a b c$ & 5769.0 & 5947.7 & $a b c$ \\
\hline Line 14 & 5076.7 & cdefg & 6109.3 & 5593.0 & abcd \\
\hline Mikham-2002 & 5694.7 & abcde & 6300.0 & 5997.3 & $a b c$ \\
\hline Line 16 & 4814.7 & defg & 6305.0 & 5559.8 & abcd \\
\hline Line 17 & 5226.0 & bcdef & 6015.0 & 5620.5 & abcd \\
\hline Line 18 & 5161.3 & bcdefg & 6761.0 & 5961.2 & $a b c$ \\
\hline Line 19 & 6583.7 & $\mathrm{a}$ & 6455.0 & 6519.3 & $\mathrm{a}$ \\
\hline Alperbey & 5853.3 & abcd & 5970.3 & 5911.8 & $a b c$ \\
\hline Line 21 & 6360.7 & $\mathrm{ab}$ & 6388.3 & 6374.5 & $\mathrm{ab}$ \\
\hline Line 22 & 6551.0 & $\mathrm{a}$ & 6430.0 & 6490.5 & $\mathrm{a}$ \\
\hline Line 23 & 5874.7 & abcd & 5939.0 & 5906.8 & $a b c$ \\
\hline Line 24 & 6265.0 & $a b c$ & 6661.0 & 6463.0 & $\mathrm{a}$ \\
\hline Karma-2000 & 4513.3 & efg & 6345.0 & 5429.2 & bcd \\
\hline Mean & \multicolumn{2}{|c|}{$5537.0 \mathrm{~b}$} & $6342.0 \mathrm{a}$ & \multicolumn{2}{|c|}{5918.4} \\
\hline $\mathrm{CV}$ & \multicolumn{2}{|c|}{13.96} & 14.95 & \multicolumn{2}{|c|}{14.56} \\
\hline LSD & \multicolumn{2}{|c|}{$1269.1^{* *}$} & 1556.4 & \multicolumn{2}{|c|}{$991.3 * *$} \\
\hline
\end{tabular}

Pn values of genotypes varied between 17.15-21.71 $\mu \mathrm{mol} \mathrm{CO} \mathrm{CO}_{2}^{-2} \mathrm{~s}^{1}$ booting stage, between 13.55-16.60 $\mu \mathrm{mol}$ $\mathrm{CO}_{2} \mathrm{~m}^{-2} \mathrm{~s}^{1}$ in anthesis and between 12.45-14.88 $\mu \mathrm{mol} \mathrm{CO}_{2}$ $\mathrm{m}^{-2} \mathrm{~s}^{1}$ grain filling periods. As the growth progresses, Pn values of genotypes decreased with leaf aging. Many researchers reported photosynthesis as one of the most sensitive processes that can be inhibited by high temperature (Boutraa et al., 2015). The primary effect of high temperature on wheat is to accelerate phenological development, which seems to be associated with premature leaf senescence (Midmore et al., 1982; AlKhatib and Paulsen, 1984). It was reported in previous studies that photosynthesis rate in bread wheat decreased by $6.1 \%$ between pre and post-anthesis (Rees et al., 1993) and decreased by $48.4 \%$ between anthesis and late-dough stage (Jiang et al., 2000). It was also indicated that net photosynthesis rate was closely related to chlorophyll loss and besides leaf aging, variations in photosynthesis rates throughout growth stages had also significant impacts on yield potential of wheat (Reynolds et al., 2000). Decreasing leaf water content initially induces stomatal closure (Pasban Eslam, 2011), imposing a decrease in $\mathrm{CO}_{2}$ supply to mesophyll cells and consequently, results in a decrease in leaf photosynthesis rates (Lawlor and Cornic, 2002). A genetic variation in this case was reported for triticale (Hura et al., 2007) and wheat (Loggini et al., 1999). Roohi et al., (2013) found that triticale had a lower reduction in photosynthetic traits under water deficit conditions than wheat and barley.

As the average of genotypes, $\mathrm{Ci} / \mathrm{Ca}$ ratio was observed as 0.568 in the first year and 0.600 in the second year. Despite the higher precipitations throughout the growing season in the first year, insufficient precipitations in grain filling period (Table 2) exerted a stress on plants and consequently plants closed their stomatas to prevent water loss and ultimately leaf $\mathrm{CO}_{2}$ diffusion was limited. Therefore, under insufficient moisture conditions, stomatal closure, decreased cell $\mathrm{CO}_{2}$ concentrations and $\mathrm{Ci} / \mathrm{Ca}$ ratios were reported by Cornic (2000). Low $\mathrm{Ci} / \mathrm{Ca}$ ratios can be considered as a plant response to drought stress under dry conditions (Feng, 1998; Erbs et al., 2009).

The decrease in mesophyll conductance is mainly resulted from the decrease in $\mathrm{Pn}$ rather than $\mathrm{Ci}$ (Allahverdiyev et al., 2015). It was reported that mesophyll conductance was mostly influenced by light (Evans et al., 1994), leaf angle (Loreto et al., 1994; Scartazza et al., 1998), drought stress (Lauteri et al., 1997), salt stress (Delfine et al., 1998; 1999) and cultivarspecific characteristics (Koç et al., 2003). Mesophyll conductance of the present study was observed as 85.46 mmol m $\mathrm{m}^{-2} \mathrm{~s}^{-1}$ in booting stage, $67.79 \mathrm{mmol} \mathrm{m}^{-2} \mathrm{~s}^{-1}$ in anthesis and $58.79 \mathrm{mmol} \mathrm{m}^{-2} \mathrm{~s}^{-1}$ grain filling stage. 
Flag leaf TE values varied between 2.777-3.945 mmol $\mathrm{mol}^{-1}$ at booting stage, between 2.441-3.234 mmol mol ${ }^{-1}$ at anthesis stage and between $2.926-3.984 \mathrm{mmol} \mathrm{mol}^{-1}$ at grain filling stage. Motzo et al. (2013) indicated that before anthesis the average leaf TE was higher in irrigated treatment than in rainfed one, but differences disappeared after anthesis. Species were not different with regard to average of water treatments, although a higher leaf TE was observed in triticale compared to durum wheat in rainfed treatment after anthesis.

According to Kroonenberg (1995), the fundamental patterns among the traits should be captured by biplots. In GT biplot, a vector is drawn from the biplot origin to each marker of the traits to facilitate visualization of the relationships between and among the traits (Rubio et al., 2004; Akçura, 2011). Since the cosine of the angle between the vectors of any two traits approximates the correlation coefficient between them, this view of the biplot is best for visualizing the interrelationship among the traits (Yan and Kang, 2003). The GT biplot for each of three stages (booting, anthesis and grain filling stages) explained $76 \%, 69 \%$ to $63 \%$ of the total variation of the standardized data (Figures 1, 2 and 3).

For booting stage, the largest variation explained by biplot was for Pn and gs (Figure 1). While there were significant positive correlations between grain yield and Pn-Mc-TE; between $\mathrm{Tl}$ and $\mathrm{Tr}-\mathrm{Ci} / \mathrm{Ca}(\mathrm{P}<0.01)$ in this stage, there were significant negative correlations between grain yield and Tl-Tr and between $\mathrm{Tl}$ and Pn-Mc-TE (Table 8; Figure 1). There were also significant positive correlations between $\mathrm{Tr}$ and gs-Ci/Ca and significant negative correlations were observed between $\mathrm{Pn}, \mathrm{Mc}$ and TE (Figure 1). Tavakoli et al. (2011) reported a positive correlation between gs and transpiration rate. The correlation between photosynthesis and stomatal conductance showed that the stomatal limitation was more important than non-stomatal limitation. The decrease in transpiration rate per leaf area under stress was related to stomatal closure and such a closure decreased stomatal conductance (Abdoli and Saeidi, 2013). While there were significant positive correlations between stomatal conductance (gs) and $\mathrm{Pn}-\mathrm{Ci} / \mathrm{Ca}$, between $\mathrm{Pn}$ and $\mathrm{Mc}-\mathrm{TE}$ and between $\mathrm{Mc}$ and TE, significant negative correlations were observed between $\mathrm{Ci} / \mathrm{Ca}$ ratio and Mc-TE (Figure 1). Throughout the vegetative and generative stages, a positive correlation was also reported between Pn and gs of triticale (Hura et al., 2007). Koç et al. (2003) and Del Pozo et al. (2005) also reported significant correlations between gs and $\mathrm{Ci} / \mathrm{Ca}$ ratio.

Table 8. Correlation coefficients for physiological traits during booting, anthesis and grain filling stages

\begin{tabular}{|c|c|c|c|c|c|c|c|c|}
\hline Stage & Traits & GY & Tl & $\mathbf{T r}$ & gs & Pn & $\mathrm{Ci} / \mathrm{Ca}$ & Mc \\
\hline \multirow{7}{*}{ Booting } & $\mathrm{Tl}$ & $-0.33 * *$ & & & & & & \\
\hline & $\operatorname{Tr}$ & $-0.18 *$ & $0.89 * *$ & & & & & \\
\hline & gs & 0.14 & 0.13 & $0.49 * *$ & & & & \\
\hline & Pn & $0.30 * *$ & $-0.54 * *$ & $-0.23 * *$ & $0.46^{* *}$ & & & \\
\hline & $\mathrm{Ci} / \mathrm{Ca}$ & 0.03 & $0.32 * *$ & $0.52 * *$ & $0.69 * *$ & -0.15 & & \\
\hline & Mc & $0.22 * *$ & $-0.55 * *$ & $-0.42 * *$ & -0.02 & $0.82 * *$ & $-0.66 * *$ & \\
\hline & $\mathrm{TE}$ & $0.33 * *$ & $-0.92 * *$ & $-0.81 * *$ & -0.12 & $0.66 * *$ & $-0.45 * *$ & $0.71 * *$ \\
\hline \multirow{7}{*}{ Anthesis } & $\mathrm{Tl}$ & $-0.34 * *$ & & & & & & \\
\hline & $\operatorname{Tr}$ & $-0.30 * *$ & $0.71 * *$ & & & & & \\
\hline & gs & -0.11 & 0.08 & $0.71 * *$ & & & & \\
\hline & Pn & $-0.16^{*}$ & $0.25 * *$ & $0.64 * *$ & $0.65 * *$ & & & \\
\hline & $\mathrm{Ci} / \mathrm{Ca}$ & 0.06 & $-0.25 * *$ & $0.18^{*}$ & $0.52 * *$ & $-0.25 * *$ & & \\
\hline & $\mathrm{Mc}$ & -0.15 & $0.37 * *$ & $0.42 * *$ & $0.22 * *$ & $0.78 * *$ & $-0.59 * *$ & \\
\hline & $\mathrm{TE}$ & $0.27 * *$ & $-0.70 * *$ & $-0.72 * *$ & $-0.37 *$ & 0.03 & $-0.49 * *$ & 0.13 \\
\hline \multirow{7}{*}{$\begin{array}{l}\text { Grain } \\
\text { Filling }\end{array}$} & $\mathrm{Tl}$ & -0.14 & & & & & & \\
\hline & $\operatorname{Tr}$ & 0.08 & $0.69 * *$ & & & & & \\
\hline & gs & $0.31 * *$ & $-0.40 * *$ & $0.33 * *$ & & & & \\
\hline & Pn & 0.12 & $-0.44 * *$ & 0.03 & $0.60 * *$ & & & \\
\hline & $\mathrm{Ci} / \mathrm{Ca}$ & $0.28 * *$ & $-0.31 * *$ & $0.29 * *$ & $0.74 * *$ & 0.03 & & \\
\hline & $\mathrm{Mc}$ & -0.07 & 0.03 & -0.11 & -0.13 & $0.59 * *$ & $-0.72 * *$ & \\
\hline & TE & 0.00 & $-0.81 * *$ & $-0.78 * *$ & 0.07 & $0.56 * *$ & $-0.23 * *$ & $0.43^{* *}$ \\
\hline
\end{tabular}

$*$ and $* *$ mean significant difference at 0.05 and 0.01 , respectively.

For anthesis stage, the largest variation explained by biplot was for $\mathrm{Ci} / \mathrm{Ca}$, TE, $\mathrm{Tr}$ (Figure 2). While there were significant positive correlations between grain yield and TE in this stage, negative correlations were observed between grain yield and the other physiological traits (Table 8; Figure 2). While significant positive correlations were observed between $\mathrm{Tl}$ and $\mathrm{Tr}-\mathrm{Pn}-\mathrm{Mc}(\mathrm{P}<0.01)$, there were significant negative correlations between $\mathrm{Ci} / \mathrm{Ca}$ and TE (Figure 2). Significant positive correlations were also observed between $\mathrm{Tr}$ and gs-Pn-Ci/Ca-Mc, between gs and $\mathrm{Pn}-\mathrm{Ci} / \mathrm{Ca}-\mathrm{Mc}$, between $\mathrm{Pn}$ and $\mathrm{Mc}$ and between and significant negative correlations were observed between $\mathrm{Tl}$ and $\mathrm{Ci} / \mathrm{Ca}-\mathrm{TE}$, between $\mathrm{Tr}$ and TE, between gs and TE, 
between $\mathrm{Pn}$ and $\mathrm{Ci} / \mathrm{Ca}$ and finally between $\mathrm{Ci} / \mathrm{Ca}$ and $\mathrm{Mc}-\quad \mathrm{TE}$.

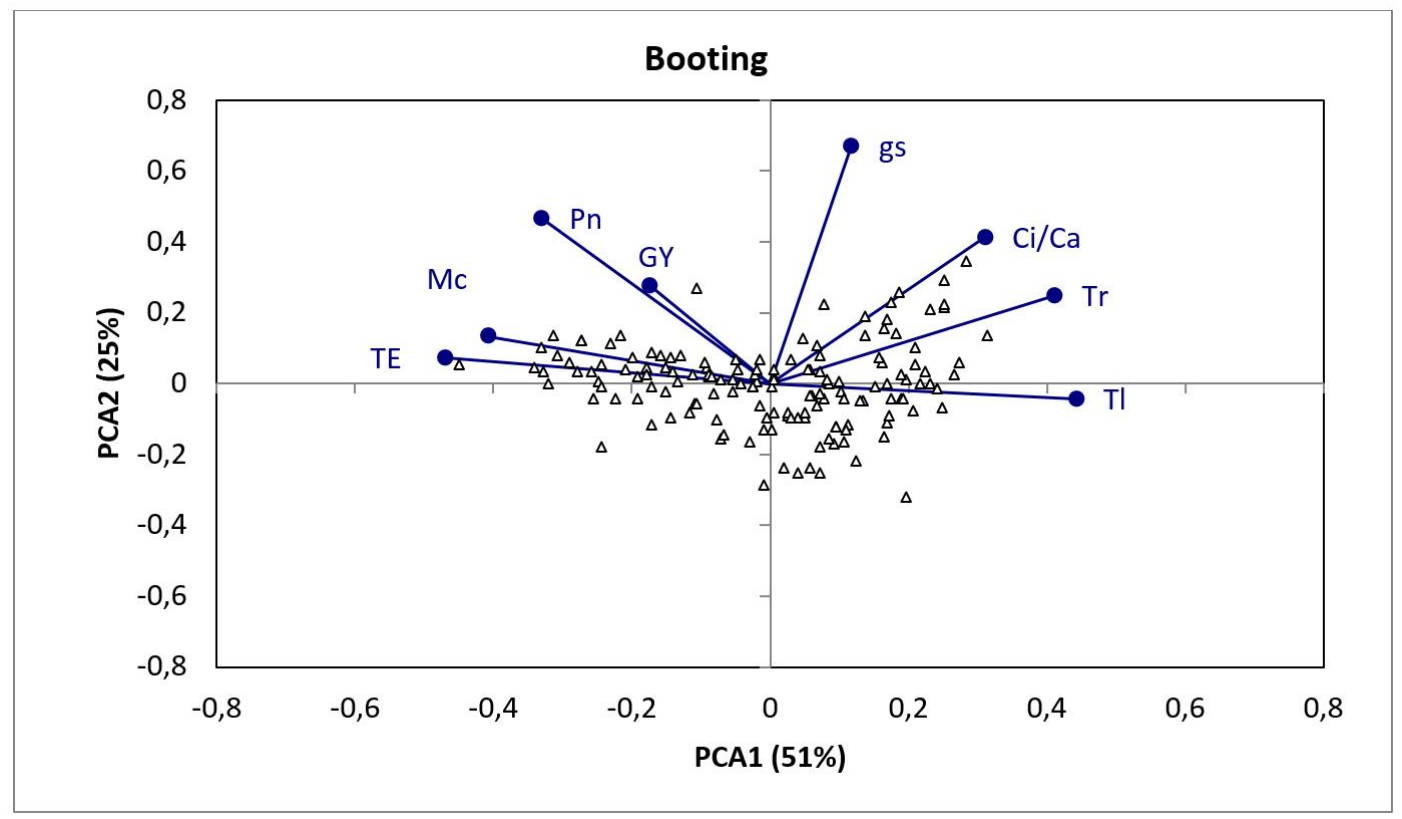

Figure 1. Relationships between physiological traits of triticale genotypes at booting stage $(n=150)$

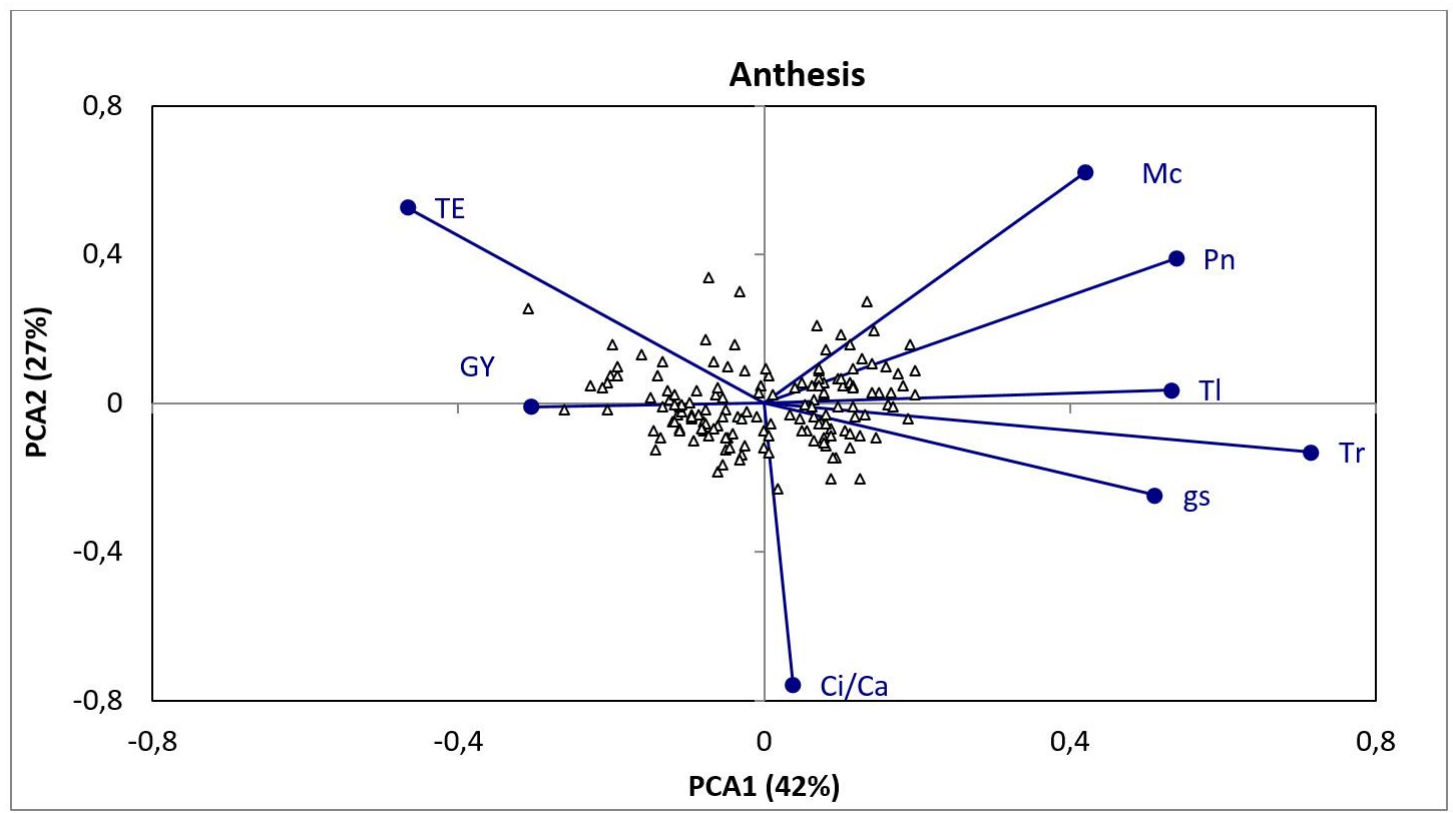

Figure 2. Relationships between physiological traits of triticale genotypes at anthesis stage $(n=150)$

For grain filling stage, the largest variation explained by biplot was for TI, Tr, and TE (Figure 3). While there were significant positive correlations between grain yield and $\mathrm{gs}-\mathrm{Ci} / \mathrm{Ca} \quad(\mathrm{P}<0.01)$ in this stage, the correlations between grain yield and the other physiological traits were not found to be significant (Table 8, Figure 3). Significant positive correlations were also observed between $\mathrm{Tl}$ and $\mathrm{Tr}$, between $\mathrm{Tr}$ and gs-Ci/Ca, between gs and $\mathrm{Pn}-\mathrm{Ci} / \mathrm{Ca}$, between Pn and Mc-TE and between Mc and TE and significant negative correlations were observed between $\mathrm{Tl}$ and gs-Pn-Ci/Ca-TE, between $\mathrm{Tr}$ and $\mathrm{TE}$ and finally between $\mathrm{Ci} / \mathrm{Ca}$ and Mc-TE (Figure 3). Hui et al. (2008) reported significant positive correlations between $\operatorname{Tr}$ and
$\mathrm{Tl}$ in late grain filling stage of wheat. Fischer et al. (1998) studied the yield of wheat associated with gs and Pn, and concluded that gs may be potential indirect selection criteria for yield.

Delgado et al. (1994) and Monneveux et al. (2006) indicated a significant correlation between grain yield and net photosynthesis rate in wheat and Fischer et al. (1981) indicated the same correlation as insignificant in wheat. Rees et al. (1993) reported positive correlations between grain yield and photosynthesis rate in pre and post anthesis stages and Reynolds et al. (2000) indicated significant correlations between net photosynthesis rate 
and grain yield at 3 growth stages (booting, anthesis and grain filling).

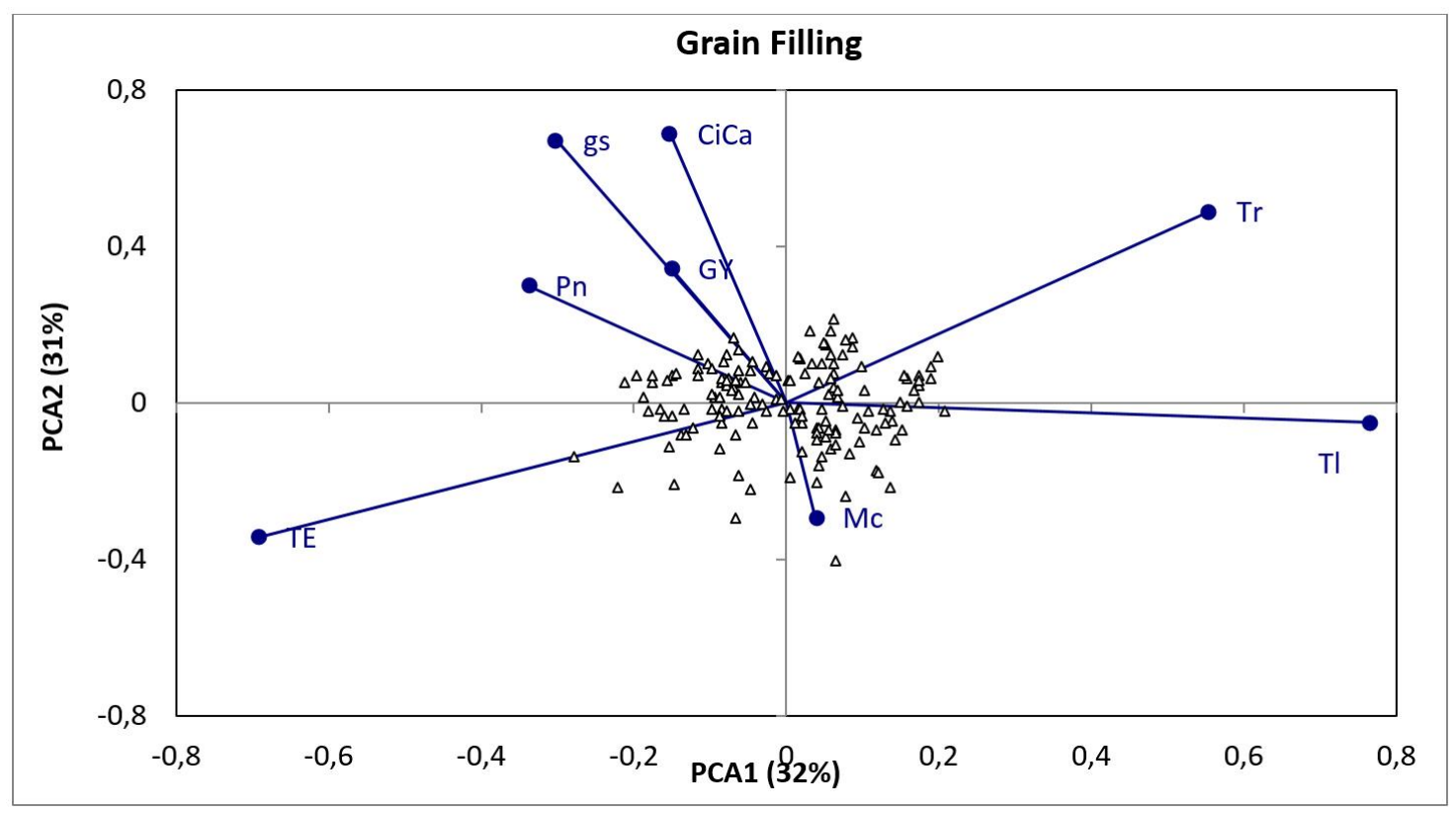

Figure 3. Relationships between physiological traits of triticale genotypes at grain filling stage $(n=150)$.

There were highly positive correlations between stomatal conductance (gs) and $\mathrm{Tr}$ for all three stages (Table 8). The level of correlation between gs and TE decreased with increasing drought stress in grain filling period. Such a case then resulted in decreasing gs values through weakened flag leaf functions because of high leaf temperature (Shao et al. 2005; Tan et al., 2006).

The negative correlation between leaf temperature (TI) and TE was observed for all 3 stages. Feng et al. (2005) indicated that there was always a negative correlation between $\mathrm{Tl}$ and $\mathrm{TE}$ and such a correlation was stronger especially in late grain filling stage. Inefficient water use throughout late ripening stage because of increasing temperatures was indicated as a disadvantage for wheat (Shao et al. 2005).

A negative correlation was reported between net photosynthesis rate $(\mathrm{Pn})$ and $\mathrm{Ci} / \mathrm{Ca}$ ratio (Del Pozo et al., 2005) and significant positive correlations were reported between Mc and Pn (Fischer et al., 1998; Del Blanco et al., 2000). Correlation analyses revealed the dominant role of $\mathrm{Mc}$ in regulation of Pn. Such a finding is in agreement with the result of Siddique et al., (1999).

\section{CONCLUSIONS}

The relationships between grain yield and physiological parameters of triticale were investigated in this study. Physiological parameters positively correlated with grain yield varied for three growth stages. Therefore, results of each stage should be assessed separately. It is recommended for further triticale breeding studies that the genotypes with high $\mathrm{Pn}, \mathrm{TE}$ and $\mathrm{Mc}$ values at booting stage, high TE values at anthesis stage and high gs and $\mathrm{Ci} / \mathrm{Ca}$ values at grain filling stage should be investigated. On the other hand, the traits of $\mathrm{Tl}$ and $\mathrm{Tr}$ with significant stable negative correlations with grain yield could also be used for selections. The results of this research showed that the selection of triticale genetic materials with highphotosynthesis (Pn), high-tranpiration efficiency (TE) low-transpiration (Tr) and low- leaf temperature (Tl) during all stages. According to this results, Line 1, Line 2, Line 3, Line 5, Line 7, Line 13 and Line 22 genotypes can be investigated for physiological traits for further breeding.

\section{LITERATURE CITED}

Abdoli M. and M. Saeidi. 2013. Evaluation of water deficiency at the post anthesis and source limitation during grain filling on grain yield, yield formation, some morphological and phonological traits and gas exchange of bread wheat cultivar. Albanian J. of Agricultural Sciences 12(2), 255-265.

Akçura, M. 2011. The relationships of some traits in Turkish winter bread wheat landraces. Turkish J. of Agriculture and Forestry, 35(2), 115-125.

Al-Khatib, K. and G.M., Paulsen. 1984. Mode of high temperature injury to wheat during grain development. Physiol. Plant 61, p.363-368.

Allahverdiyev, T.I., J.M. Talai, I.M. Huseynova and J.A., Aliyev. 2015. Effect of drought stress on some physiological parameters, yield, yield components of durum (Triticum durum desf.) and bread (Triticum aestivum L.) wheat genotypes. Ekin Journal of Crop Breeding and Genetics, 1-1; 50-62.

Azhand, M., M. Saeidi and M. Abdoli. 2015. Evaluation of the relationship between gas exchange variables with grain yield in barley genotypes under terminal drought stress. International J. of Biosciences, 6-2; 366-374.

Bassu, S., S. Assengb and R. Richards. 2011. Yield benefits of triticale traits for wheat under current and future climates. Field Crops Research, 124, 14-24.

Bilgili, U., E.A. Cifci, H. Hanoglu, K. Yagdi and E. Acikgoz. 2009. Yield and quality of triticale forage. J. Food Agric. Environ. 7, 556-560. 
Boutraa, T., A. Akhkha and A.K. Al-Shoaibi. 2015. Evaluation of growth and gas exchange rates of two local saudi wheat cultivars grown under heat stress conditions. Pakistan J. of Botany, 47(1):27-34.

Bunce, J.A. 1998. The Temperature dependence of the stimulation of photosynthesis by elevated carbon dioxide in wheat and barley, Journal of Experimental Botany, 49(326): $1555-1561$.

Cornic, G. 2000. Drought stress inhibits photosynthesis by decreased stomatal aperture-not by affecting ATP synthesis. Trends in Plant Science, 5:187-188.

Del Blanco, I.A., Rajaram, S., Kronstad, W.E., Reynolds, M.P. 2000. Physiological performnce of synthetic hexaploid wheat-derived populations. Crop Sci., 40(5):1257-1263.

Delfine, S., Alvino, A., Zacchini, M., Loreto, F. 1998. Consequences of salt stres on conductance to $\mathrm{CO}_{2}$ diffusion, rubisco characteristics and anatomy of spinach leaves. Australian Journal of Plant Physiology. 25:395-402.

Delfine, S., Alvino, A., Concetta-Villani, M., Loreto, F. 1999. Restriction to carbon dioxide conductance and photosynthesis in spinach leaves recovering from salt stres. Plant Physiology. 119:1101-1106.

Delgado, M.I., Reynolds, M.P., Larque-Saavedra, A., Nava, S.T. 1994. Genetic diversity for photosynthesis in wheat under heat stresssed field environments and its relation to productivity. Wheat Special Report, No.30, 17 pages, Mexico.

Del Pozo, A., Perez, P., Morcuende, R., Alanso, A., MartinezCarrasco, R. 2005. Acclimatory responses of stomatal conductance snd photosynthesis to elevated $\mathrm{CO}_{2}$ and temperature in wheat crops grown at varying levels of $n$ supply in a mediterranean environment. Plant Science, 169:908-916.

Erbs, M., Franzaring, J., Hogy, P., Fangmeir, A. 2009. Free-air $\mathrm{CO}_{2}$ enrichment in a wheat-weed assembly-effects on water relations. Basic and Applied Ecology, 10:358-367.

Evans, L.T., S. Von Caemmerer, B.A. Stetchell and G.S. Hudson. 1994. The relationship between $\mathrm{CO}_{2}$ transfer conductance and leaf anatomy in transgenic tobacco with a reduced content of Rubisco. Australian J. of Plant Physiology, 21:475-495.

Faostat Database,

2010. http://faostat.fao.org/site/567/default.aspx\#ancor.

Feng, X., 1998. Long term $\mathrm{Ci} / \mathrm{Ca}$ response of trees in western North America to atmospheric $\mathrm{CO}_{2}$ concentration derived from carbon isotope chronologies. Oecologia, $117: 19-25$.

Feng, B.L., X.L. Gao, L. Zhao, J.F. Gao, C.F. Wang and S.W. Zhang. 2005. Relationships between canopy temperature and biological characters of wheat under drought conditions. Chin J. Ecol. 24 (5), 508-512 (in Chinese).

Fischer, R.A., F. Bidinger, J.R. Syme and P.C. Wall. 1981. Leaf photosynthesis, leaf permeability, crop growth, and yield of short spring wheat genotypes under irrigation. Crop Sci., 21(3): 367-373.

Fischer, R.A., D. Rees, K.D. Sayre, Z.M. Lu, A.G. Condon and A. Larque-Saavedra. 1998. Wheat yield progress associated with higher stomatal conductance and photosynthetic rate, and cooler canopies. Crop Sci., 38 (6): 1467-1475.

Giunta, F., R. Motzo and G. Pruneddu. 2003. Comparison of temperate cereals and grain legumes in a Mediterranean environment. Agr. Med. 133, 234-248.

Giunta, F., R. Motzo and A. Virdis. 2001. Development of durum wheat and triticale cultivars as affected by thermophotoperiodic conditions. Austr. J. Agric. Res. 52, 387-396.

Gowda, M., V. Hahn, J.C. Reif, C.F.H. Longin, K. Alheit and H.P. Maurer. 2011. Potential for simultaneous improvement of grain and biomass yield in Central European winter triticale germplasm. Field Crops Research, 121, 153-157.

Harding, S.A., J.A. Guikema and G.M. Paulsen. 1990. Photosynthetic decline from high temperature stress during maturation of wheat. I. Interaction with senescence process. Plant Physiol. 92, 648-653.

Hura, T., K. Hura, M.T. Grzesiak and K. Rzepka. 2007. Effect of long-term drought stress on leaf gas Exchange and fluorescence parameters in $\mathrm{C} 3$ and $\mathrm{C} 4$ plants. Acta Physiol Plant, 29:103-113.

Hura, T., K. Hura and M.T. Grzesiak, 2009. The usefulness of the chlorophyll fluorescence parameters in harvest prediction in 10 genotypes of winter triticale under optimal growth conditions. Plant Biosystems, 143(3):496-503.

Hui, Z., Z. Zhengbin, S. Hongbo, X. Ping and M.J. Foulkes. 2008. Genetic correlation and path analysis of transpiration efficiency for wheat flag leaves. Environmental and Experimental Botany, 64(1):128-134.

Igne B, L.R. Gibson, A. Rippke and C. R. Hurburg, 2007. Triticale moisture and protein content prediction by nearinfrared pectroscopy. Cereal Chemistry, 84: 328-330.

Jiang, G.M., N.B. Hao, K.Z. Bai, Q.D. Zhang, J.Z. Sun, R.J. Guo, Q.Y. Ge and T.Y. Kuang. 2000. Chain correlation between variables of gas exchange and yield potential in different winter wheat cultivars. Photosynthetica, 38(2): 227232.

Kaplan, M., M. F. Yilmaz and R. Kara, 2015. Variation in hay yield and quality of new triticale lines. Journal of Agricultural Sciences, 21:50-60.

Koç, M., C. Barutçular and I. Genç. 2003. Photosynthesis and productivity of old and modern durum wheats in a Mediterranean environment. Crop Sci., 43(3): 2089-2098.

Kozak, M., S. Samborski, J. Rozbicki and W. Madry. 2007. Winter triticale grain yield a comparative study of 15 genotypes. Acta Agric. Scand. 57, 263-270.

Kroonenberg, P.M. 1995. Introduction to biplots for $\mathrm{G} \times \mathrm{E}$ tables. Department of Mathematics, Research Report 51, University of Queensland.

Lauteri, M., A. Scartazza, M.C. Guido and E. Brugnoli. 1997. Genetic variation in photosynthetic capacity, carbon ssotope discrimination and mesophyll conductance in provenances of Castanea sativa adapted to different environments. Functional Ecology. 11:675-683.

Lawlor D.W., and G. Cornic. 2002. Photosynthetic carbon assimilation and associated metabolism in relation to water deficits in higher plants. Plant, Cell \& Environment 25: 275 294.

Lekgari, L.A., P.S. Baenziger, K.P. Vogel and D.D. Baltensperger. 2008. Identifying winter forage triticale $(\times$ Triticosecale Wittmack) strains for the central Great Plains. Crop Sci. 48, 2040-2048.

Lipkovich I.A. and E.P. Smith. 2002. Biplot and singular value decomposition macros for Excel(C). Journal of Statistical Software, 7(5): 1-15.

Loggini B, A. Scartazza, E. Brugnoli and F. Navari-Izzo. 1999. Antioxidative defense system, pigment composition, and photosynthetic efficiency in two wheat cultivars subjected to drought. Plant Physiology, 119, 1091-1099.

Lopez-Castaneda, C. and R. Richards. 1994. Variation in temperate cereals in rainfed environments I. Grain-yield, biomass and agronomic characteristics. Field Crops Res. 37, 51-62.

Loreto, F., G. Di Marco, D. Tricoli and T.D. Sharkey. 1994. Measurement of mesophyll conductance, photosynthetic electron transport and alternative electron sinks of fieldgrown wheat leaves. Photosynthesis Research, 41,397-403. 
Midmore, D.J., P.M. Cartwright and R.A. Fischer. 1982. Wheat in tropical environments. I. Phasic development and spike size. Field Crops Research, 5,185-200.

Monneveux, P., D. Rekika, E. Acevedo and O. Merah. 2006. Effect of drought on leaf gas exchange, carbon isotope discrimination, transpiration efficiency and productivity in field grown durum wheat genotypes. Plant Sci., 170:867872.

Motzo, R., G. Pruneddu and F. Giunta. 2013. The role of stomatal conductance for water and radiation use efficiency of durum wheat and triticale in a Mediterranean environment. European J. of Agronomy, 44:87-97.

Pasban Eslam, B. 2011. Evaluation of physiological indices for 1mproving water deficit tolerance in spring safflower. J. Agr. Sci. Tech., 13,327-338.

Qiu, G.Y., L. Wang, X. He, X. Zhang, S. Chen, J. Chen and Y. Yang. 2008. Water use efficiency and evapotranspiration of winter wheat and its response to 1rrigation regime in the North China Plain. Agricultural and Forest Meteorology, $148,1848-1859$.

Rees, D., K. Sayre, E. Acevedo, T. Navas, Z. Lu, E. Zeiger and A. Limon. 1993. Canopy temperatures of wheat: relationship with yield and potential as a technique for early generation selection, Wheat Special Report No. 10, 32 Pages, Mexico.

Reynolds, M.P., B.M.I. Delgado, M. Gutierrez-Rodriguez and A. Larque -Saavedra. 2000. Photosynthesis of wheat in a warm, irrigated environment I: Genetic diversity and crop productivity. Field Crops Research, 66, 37-50.

Richards, R., M. Watt and G. Rebetzke. 2007. Physiological traits and cereal germplasm for sustainable agricultural systems. Euphytica 154,409-425.

Roohi, E., Z. Tahmasebi-Sarvestanil, S. A. M. Modarres-Sanavy and A. Siosemardeh. 2013. Comparative study on the effect of soil water stress on photosynthetic function of triticale, bread wheat, and barley. J. Agr.Sci. Tech., 15: 215-228.

Ruuska, S., G. Rebetzke, A.F. van Herwaarden, R. Richards, N Fettell, L. Tabe and C. Jenkins. 2006. Genotypic variation in water-soluble carbohydrate accumulation in wheat. Funct. Plant Biol. 33,799-809.
Rubio, J., J. I. Cubero, L. M. Martin, M. J. Suso and F. Flores. 2004. Biplot analysis of trait relations of white lupin in Spain. Euphytica, 135(2): 217-224.

Santiveri, F., C. Royo and I. Romagosa. 2004. Growth and yield responses of spring and winter triticale cultivated under Mediterranean conditions. Eur. J. Agron. 20, 281-292.

SAS Inst (1999). SAS User's Guide: Statistic. Statistical analysis systems institute Inc., Cary, NC.

Scartazza, A., M. Lauteri, M.C. Guido and E. Brugnoli, 1998. Carbon 1sotope discrimination in leaf and stem sugars, water use efficiency and mesophyll conductance during different developmental stages in rice subjected to drought. Australian Journal of Plant Physiology. 25:489-498.

Shao, H.B., Z.S. Liang, M.A. Shao, S.M. Sun and Z.M. Hu, 2005. Investigation on dynamic changes of photosynthetic characteristics of 10 wheat (Triticum aestivum L.) genotypes during two vegetative-growth stages atwater deficits. Biointerfaces 43,221-227.

Siddique MRB, A, Hamid and M.S. Islam. 1999. Drought stress effects on photosynthetic rate and leaf gas Exchange of wheat. Bot. Bull. Acad. Sin. 40:141-145.

Tan, Y., Z.S. Liang, H.B. Shao and F. Du. 2006. Effect of water deficits on the activity of anti-oxidative enzymes and osmoregulation among 3 different genotypes of Radix Astragali at seeding stage. Biointerfaces 48, 60-65.

Tavakoli A, A. Ahmadi, M. Saeidi and S. Madah Hossani. 2011. Study of water relation and gas exchange in drought tolerant and susceptible wheat (Triticum aestivum L.) cultivars under post-anthesis drought stress condition. 2nd Iranian Conference on Plant Physiology, Yazd, 124.

Tohver, M., A. Kann, R. Taht, H. Mihhalevski, J. Hakman. 2005. Quality of triticale cultivars suitable for growing and bread-making in northern conditions. Food Chem. 89, 125132.

von Caemmerer, S. and G.D. Farquhar. 1981. Some relationships between the biochemistry of photosynthesis and the gas exchange of leaves. Planta, 153:376-387.

Yan W. and M.S., Kang. 2003. GGE Biplot Analysis: A graphical tool for breeders, Geneticists, and Agronomists. CRC Press, Boca Raton, Florida, USA. 273 p. 